\title{
Characterization Of Commercial Magnetorheological Fluids At High Shear Rate: Influence Of The Gap
}

\author{
Nicola Golinelli, Andrea Spaggiari ${ }^{*}$ \\ Department of Sciences and Methods for Engineering, University of Modena and Reggio Emilia, \\ Reggio Emilia, Italy
}

\section{Abstract}

This paper reports the experimental tests on the behaviour of a commercial MR fluid at high shear rates and the effect of the gap. Three gaps were considered at multiple magnetic fields and shear rates. From an extended set of almost two hundred experimental flow curves, a set of parameters for the apparent viscosity are retrieved by using the Ostwald de Waele model for non-Newtonian fluids. It is possible to simplify the parameter correlation by making the following considerations: the consistency of the model depends only on the magnetic field, the flow index depends on the fluid type and the gap shows an important effect only at null or very low magnetic fields. This lead to a simple and useful model, especially in the design phase of a MR based product. During the off state, with no applied field, it is possible to use a standard viscous model. During the active state, with high magnetic field, a strong non-Newtonian nature becomes prevalent over the viscous one even at very high shear rate; the magnetic field dominates the apparent viscosity change, while the gap does not play any relevant role on the system behaviour. This simple assumption allows the designer to dimension the gap only considering the non-active state, as in standard viscous systems, and taking into account only the magnetic effect in the active state, where the gap does not change the proposed fluid model.

“Corresponding author: andrea.spaggiari@unimore.it 


\section{INTRODUCTION}

The behaviour and the characterization of magnetorheological fluids (MRF) at high shear rate is a topic that is only partially discussed in scientific and technical literature. The main challenge in this particular field is to obtain a correct measure from the experimental tests. The use of MRFs in modern engineering system [1,2] is already quite wide spread due to excellent controllability $[3,4]$. Considering the field of rotatory actuators MRFs are becoming more and more popular due to their outstanding properties in terms of controllable torque, fast response time, noise reduction and small dimensions compared to standard dry friction based systems, but at the moment limited to low rotational speed. In order to keep the pace with the development of electric drives capable of very high torque and rotational speed the MRF behaviour at very high shear rate must be investigated, since the viscous forces could play an important role. When the MR fluids are subjected to high shear rate, it is not trivial to separate the rheological behaviour and the magnetic one. Rheological behaviour is related to the fluid viscosity, turbulences and fluid flow, while the magnetic behaviour is affected by the intensity of the magnetic field and the materials involved in the magnetic circuit. Other important factors, which add complexity to the problem and make the data interpretation quite difficult, are the change in viscosity, due to the friction between the ferromagnetic particles [5], due to heat generation and the redistribution the particles under the centrifugal force given by the rheometer. Mazlan et al. [6,7] while studying the basic properties of MR fluids demonstrated that the relationship between the particles lead to a spatial reorganization during compressive or tensile tests. The changes in the magnetic field are due to the fluid gap modification and due to the different phase volume of the system, as expected for hard particles. A similar approach was used in other works about the so called squeeze-strengthen effect [8-17] in which many researchers demonstrates that there is a strong enhancement of the apparent yield stress of the fluid when there is a combination of shear and compression. This behaviour is due to the formation of larger particles columns, as stated by $[11,18]$ thanks to the compressive state resulting in a higher yield stress when the magnetic field is applied. This effect was thoroughly investigated in shear [17] and appears also in flow $[8,19]$, but its application in MR system needs better understanding. Other interesting consideration on the rheological behaviour of the MR fluids, especially considering the fluid- 
1 dynamics and the magneto-static of the system are investigated in [20] by using a Searle type rheometer equipped with several rotors, with square, round and lobate profiles. Ulicny [21] considers the variation in the MR fluid behaviour in presence of step changes of magnetic flux and shows that the viscous effect plays a minor role. The typical laboratory device used to test MR fluids is the magneto-rheometer and in [22] Schramm describes the factors which influence the behaviour of MR fluid inside a traditional rheometer with concentric cylinders. The first observation is that a suitable rheometer for MR fluids analysis should prevent the magnetic force from influencing the measurement, therefore the magnetic path and the materials have to be carefully designed, taking into account the temperature issue as well, as shown in [23,24]. The second important consideration is the effect of the fluid gap, which is crucial to compute the shear rate applied to the fluid. It is shown that a gap reduction reduces the non-linearity inside the fluid gap and makes the shear rate computation more precise from the rheological standpoint. In case of thin gaps or slow shear rate the MR fluid stays in a stable - laminar condition, while in case of high shear rates Taylor vortex and instability phenomena may arise inside the gap, leading to less precise measures, as shown by Guth and Maas in [25]. They also show the potential engineering application of MRFs at high shear rate (up to $34000 \mathrm{~s}^{-1}$ ). In keeping with the previous works, the present research aims at characterizing experimentally the behaviour of a commercial MR fluid at high shear rates, with particular emphasis on the influence of different gaps and at developing a reliable and efficient model to describe their behaviour in real engineering application. The model developed, adapted on the Ostwald de Waele reference model, is very simple to be applied, since it shows that the flow curves collapses on a single one for the three gaps considered if the magnetic field is present, which simplifies the magneto-mechanical design of the system.

\section{MATERIALS AND METHODS}

Magnetorheological fluids are a suspension of ferromagnetic particles in a non-magnetic carrier fluid [26]. When there is no magnetic field applied, the magnetic particles are randomly dispersed in the fluid and the behaviour is slightly non-Newtonian. When a magnetic field is applied, the particles behaves like magnetic dipoles and align along the magnetic flux lines, forming chains able to resist to the shear 
stress up to an apparent yield stress, called $\tau_{y}$. The yield stress is a function of the magnetic field intensity.

When the external shear stress applied to the fluid is below the threshold stress $\tau_{y}$, the fluid sustains the external load like a solid. When the external stress exceeds $\tau_{y}$, the fluid starts to flow again, typically with a shear thinning behaviour. One of the best rheological model with which to describes the fluid behaviour is the Hershel-Bulkley model [27], namely:

$$
\tau=\tau_{y}+K \dot{\gamma}^{n}
$$

$$
\text { Where } \tau_{y} \text { is the yield stress, function of the magnetic induction, } K \text { is the consistency, } \dot{\gamma} \text { is the shear }
$$
rate and $n$ is the flow index. The fluid we consider is the MRF 132-DG from Lord Corporation [28]. This particular formulation uses a synthetic oil as a carrier, with nearly $80 \%$ weight content of ferromagnetic particles. The producer datasheet reports a yield stress around $50 \mathrm{kPa}$ when the magnetic field is above $300 \mathrm{KA} / \mathrm{m}$. Although it is not specified in the producer TDS we can find some information in literature work [8-17] about the average dimension of the spherical MR particles, which is around $20 \mu \mathrm{m}$, with a density around $2950-3150 \mathrm{~kg} / \mathrm{m}^{3}$. The settling and the agglomeration of the particles is prevented thanks to proprietary surfactants and additives. We decided to investigate the rheological properties of this particular fluid since the industrial applications of MR fluids are always based on commercially available MRFs. Even though he results are presented for the Lord MRF 132-DG, the proposed methodology has a general applicability, provided that the parameters of each particular fluid are retrieved by specific experimental tests, following the procedure described in this work.

\subsection{Searle Magnetorheometer}

Searle magnetorheometer is a particular rheometer design where the rotor spins and the fluid is sheared between it and a fixed housing. The rotor transfers the torque to the stator through the fluid under test. The rotation speed is measured through an encoder/resolver system and the transmitted torque is measured through a sensor that connects the housing to the ground. The main component of the Searle magnetorheometer are described in Figure 1a, namely: the housing, the rotor, the coils, the bearings and the top cover. The MR fluid reservoir and the rotor are placed inside the housing and supported by the 
1 bearings, the rotor is linked through an elastic joint to a motor and shears the MR fluid in the gaps. The

2 magnetic system is a single coil made in AWG24 with 530 turns, and it is equipped with a thermocouple

3 to avoid dangerous over-temperature problems. There are three small holes in the top cover in order to

4 connect the coil and the sensor to the power electronic outside the magnetorheometer. The two bearings are mounted onto the housing, with a slight mechanical interference. These bearings are coupled to the rotor and allows the system to spin with a very good precision around its axis. The radial eccentricity was measured with a mechanical comparator and is less than $0.02 \mathrm{~mm}$, therefore the gap height is nearly constant. Once the fluid is poured in the system and the rotor is placed the top cover is fixed to the housing granting a sealing of the parts and a closed magnetic flux path, as described in the following section. Figure $1 \mathrm{~b}$ shows the main geometrical parameters of the system, i.e. the rotor dimensions and the gap height. Since the aim of the work is to evaluate the influence of the gap on the MR fluid properties, we used three different rotors with three different $\mathrm{R}_{\mathrm{R}}$ dimensions: $R_{R}=8,8.5$ and $8.9 \mathrm{~mm}$ and maintained the external radius of the stator constant $\left(R_{S}=9 \mathrm{~mm}\right)$.

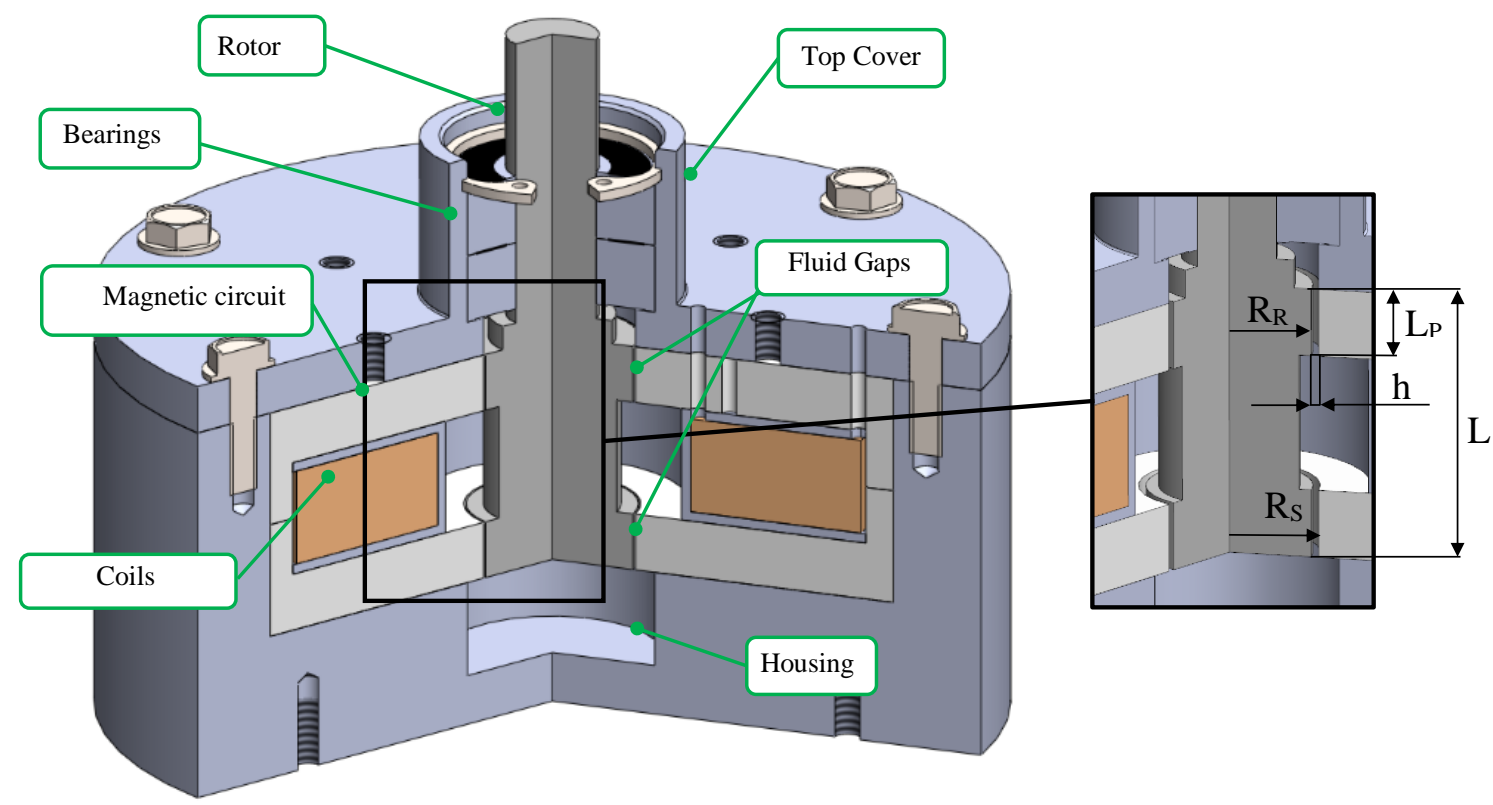

(a)

(b)

Figure 1. Partial section view of the Searle magnetorheometer (a) and main dimensions (b). 
Therefore, we have three difference fluid gaps: $h=R_{S}-R_{R}=1,0.5,0.1 \mathrm{~mm}$. We decided to investigate

9

$$
\tau=\frac{T}{2 \pi L_{P}\left(R_{R}+h\right)^{2}}
$$

$$
\dot{\gamma}=\frac{R_{R}}{h} \omega
$$

where $T$ is the torque, and $h$ is the gap height. Using the same notation and considering the angular rotation speed $\omega$ it is possible to express the shear rate, $\dot{\gamma}$, on the external rotor surface:

Where the upper group equals to the linear speed onto the rotor surface. We are aware that the equation (3) is an approximation, since the shear rate decreases linearly from its maximum value on the rotor surface down to zero onto the stator surface. Nevertheless when the gap are quite thin [30] this approximation is lead to negligible errors. As reported in literature [31,32], very refined methods are available to correct the measurement error due to three main reasons: gap error, confinement and slip. The gap error is due to eccentricity and only in case of $0.1 \mathrm{~mm}$ gap the effect could be relevant. Nevertheless, the main findings are due to differential behaviour of the MR fluid with and without magnetic field, which is not dependent on the possible error on the thinnest gap. Moreover, the eccentricity lead to a local gap variation, but the gap dimension computed on the entire rotor profile is on average at the nominal value, since the gap errors self-compensate. The confinement problem occurs only when the gap is about five times the characteristic dimension of the included phase [33], which is 
not the case for the considered MR fluid. The third problem is the MR fluid slip, as the rheometer surfaces are smooth and in absence of magnetic field the fluid could slip [31,34].

\subsection{Magnetic simulations}

Since we have three gap heights, it is necessary to evaluate the magnetic field inside the gap for each gap. It is not enough to apply the same current to the coils since the reluctance of the magnetic circuit changes and therefore a magnetic simulation of the system is needed. The aim is to find the current that produces the same induction in the three gaps. We used the free software FEMM 4.2 [35] which allows us to perform an axisymmetric simulation of the magnetorheometer. Figure 2 reports the results of one the simulations in terms of induction of magnetic field in the gap. It is immediate to notice that the flux lines are confined in some portions of the system, which are made of ferromagnetic materials (rotor shaft and magnetic path), such as mild steel. The other parts of the magnetorheometer are made of aluminium (housing and top cover) which is diamagnetic and prevents the flux to be dispersed.

We reported in Figure 3 the effect of the gap height in term of correlation between applied current and induction field obtained from the simulations and it is immediate to notice that is not possible to compare the same current when the gap is different, due to the change in the magnetic field. In order to reach the same value of magnetic field as the gap increase, it is needed a larger current value, dependent on the magnetic circuit, since the magnetic permeability of the MR fluid is considerably lower than the steel one. The interpolation of the points in Figure 3 is used in the experimental tests to tune the applied current for each gap and to obtain the desired magnetic field. It is important to highlight the strong increase in the magnetic field, for a given current, when the gap is $0.1 \mathrm{~mm}$, which means that the performances of a system with a gap this low would be very high.

\subsection{Experimental Test apparatus}

Figure 4 depicts the experimental test rig used for all the magnetorheological tests. The torque sensor is mounted on a rigid frame and supports the housing of the magnetorheometer, which is already filled the MR fluid. Afterwards, the electric direct drive motor is connected to the rotor shaft by means of an elastic joint. The power electronic used for the coils is able to inject up to 3 Amps and this causes an 
1 important heating of the system. Therefore, a cooling system is needed to maintain the coils at the desired

2 temperature. The cooling system is able to maintain the system at $25^{\circ} \mathrm{C}$ by circulating the refrigerator

3 fluid in the copper piping showed in Figure 4.

4 The maximum speed of the magnetorheometer for each experimental test is reached with a step-by-

5 step approach. Eight constant shear rate steps are needed to reach the maximum prescribed speed. The

6 shear rate and the shear stress are measured at each step in order to improve the precision of the lower

7 curves. The tangential linear velocities of the external surface of the rotor used in the experiments are:

$80.5,0.8,1.1,1.6,2.1,2.3,2.5 \mathrm{~m} / \mathrm{s}$. These linear velocities lead to a different shear rates according to the

9 three gaps height (See eq. 3). The torque signal is processed using a dedicated software in LABVIEW

10 environment, after a signal conditioner (TM-02). The prescribed current is turned on at the beginning of

11 the test so all the different steps are under the same magnetic condition.
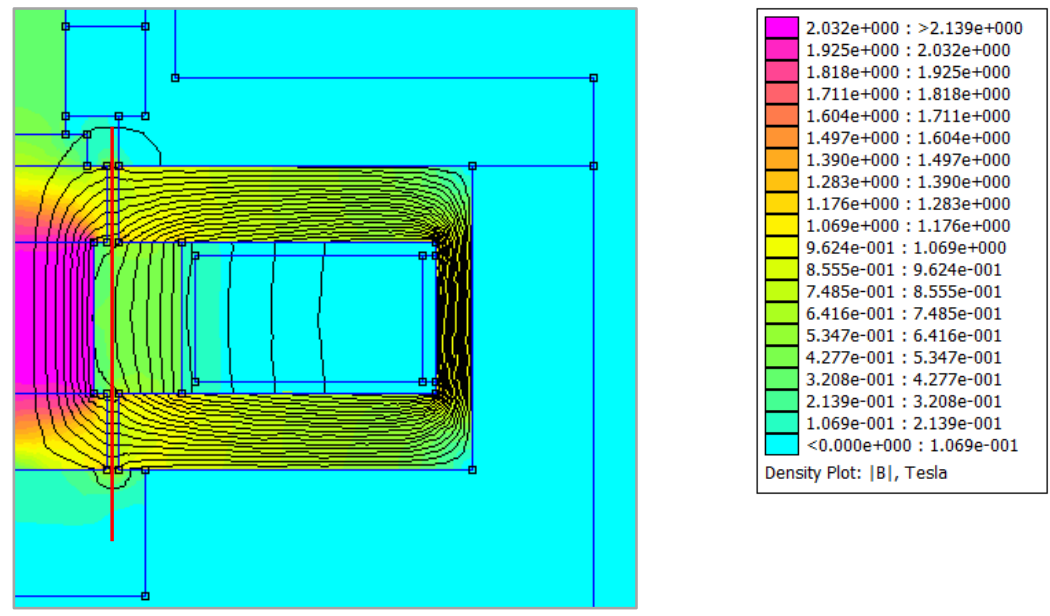

Figure 2. Flux induction lines inside the magnetorheometer $(h=1 \mathrm{~mm}, I=2.2 \mathrm{~A})$. 


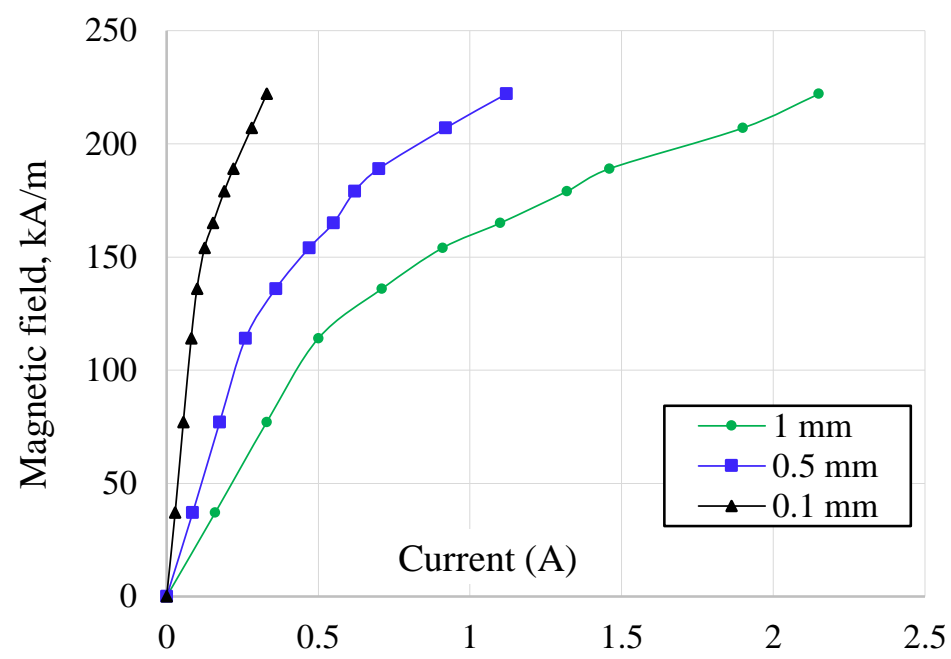

Figure 3 - Applied current vs simulated magnetic field for the three gaps considered.

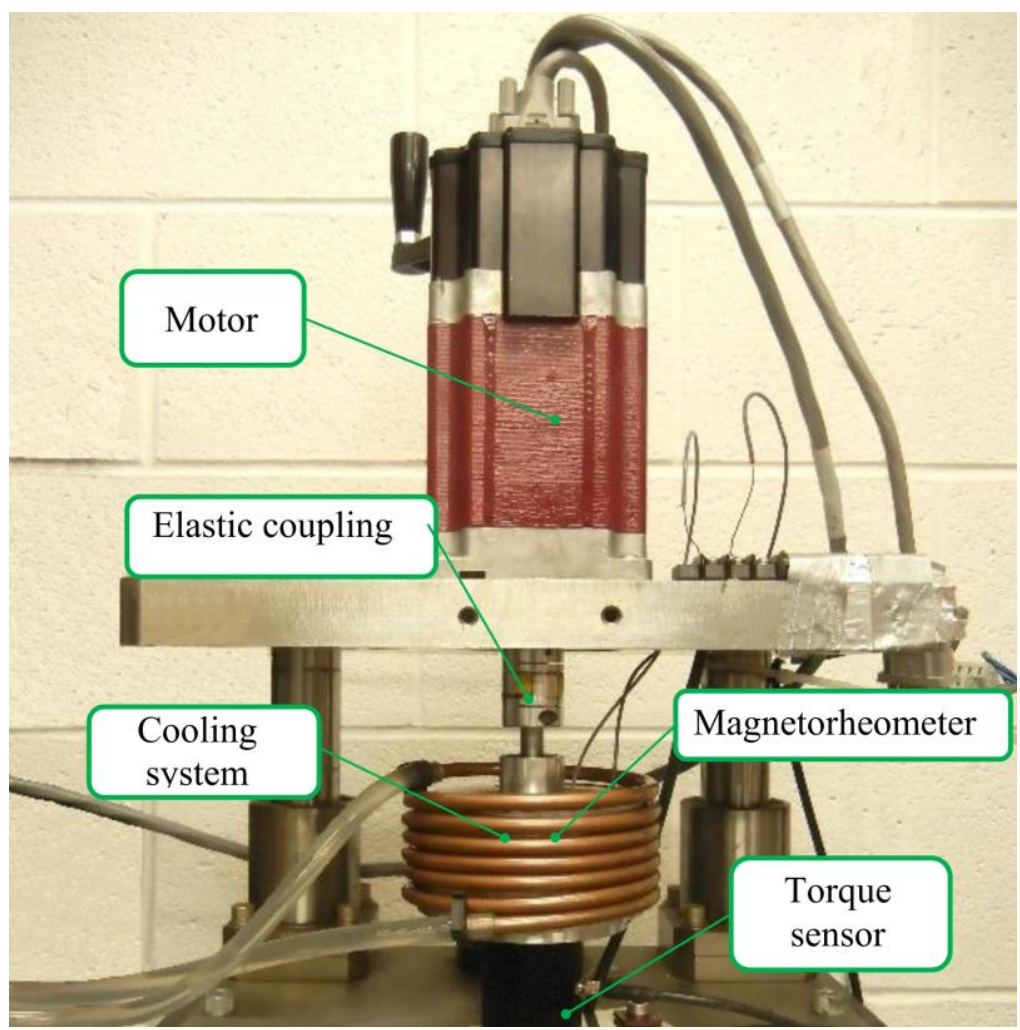

Figure 4. Experimental set-up, with cooling system 


\section{RESULTS}

According to the data collected in the experimental tests, it was possible to build the typical flow curves to study the behaviour of fluids. This leads to three levels of maximum shear rate for each gap thickness: in particular $2500 \mathrm{~s}^{-1}$ with $h=1 \mathrm{~mm}, 5000 \mathrm{~s}^{-1}$ with $h=0.5 \mathrm{~mm}$ and $25000 \mathrm{~s}^{-1}$ with $h=0.1$ $m m$. Figure 5a-c-e reports the flow curves for each gap used. An additional feature that is interesting to evaluate is the apparent viscosity of the fluid, $\eta$, which is related, such as the shear stress, to the shear rate. The apparent viscosity was calculated, by simply dividing the shear stress $\tau$ by the respective shear rate $\dot{\gamma}$. MR fluids, which belong to a sub-category of pseudoplastic fluids, show a decrease in apparent viscosity as the velocity gradient increases, as can be seen from the graphs in Figure 5b-d-f. An immediate qualitative data analysis shows a flattening of the flow curves, especially in the post-yielding phase in the fluid, which makes shear stress $\tau$ only slightly dependent on the shear rate. Goncalves [36] noticed the same effect, but due to exposure time of the MR fluid to the magnetic field. The apparent viscosity gradually decreases as the shear rate increases, as expected, but there is a noticeable collapse of the curves at different magnetic fields only for the lower gaps. Figure 6a depicts in a Log-Log chart the apparent viscosity for all the gaps. Each magnetic field is represented by a different colour. Figure $6 \mathrm{~b}$ represents only the curve with a magnetic field above $77 \mathrm{KA} / \mathrm{m}$ a threshold value above which it is nearly impossible to discriminate the different gaps. i.e. the three curves of the same colours are superimposed.

\section{DISCUSSION}

The time needed to achieve the equilibrium between the applied shear rate and the correspondent shear stress is much lower as low viscosity is. This leads to a short time to reach equilibrium when the shear rate is high while a longer test is needed when $\dot{\gamma}$ is low. The evidence of this phenomenon is an increase of the shear stress with increasing shear rate below $1000 \mathrm{~s}^{-1}$ as can be seen in Figure 5a,c. In addition, due to partial residual magnetization of the rotor, it is possible to hypothesize a slight residual activation of the fluid which could influence the measured torque especially at the slow speed [37]. 


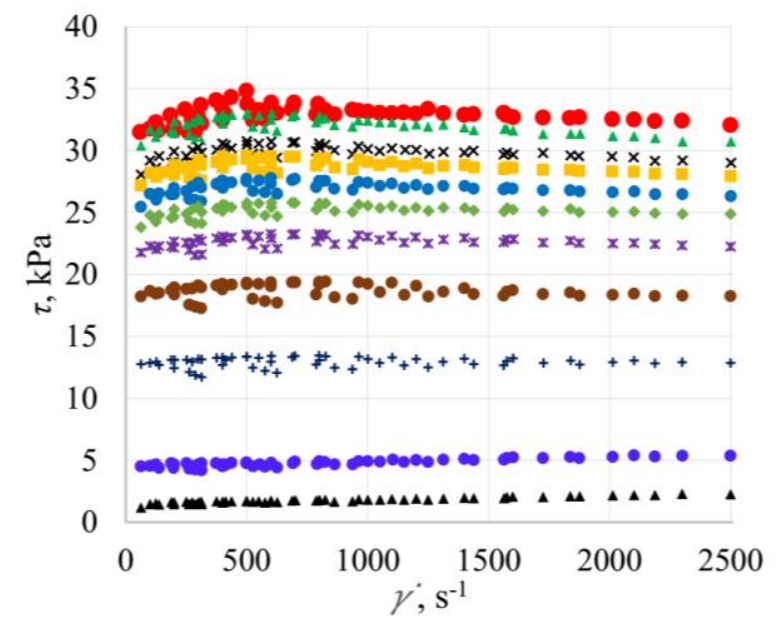

(a)

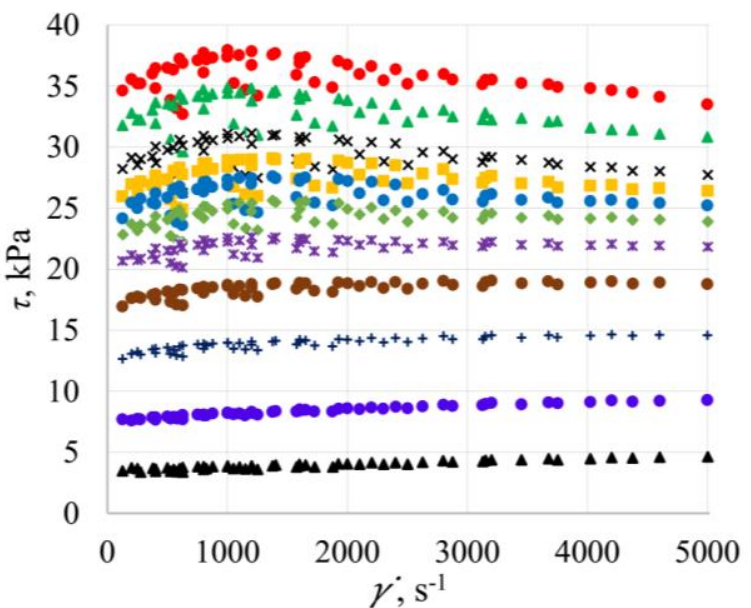

(c)

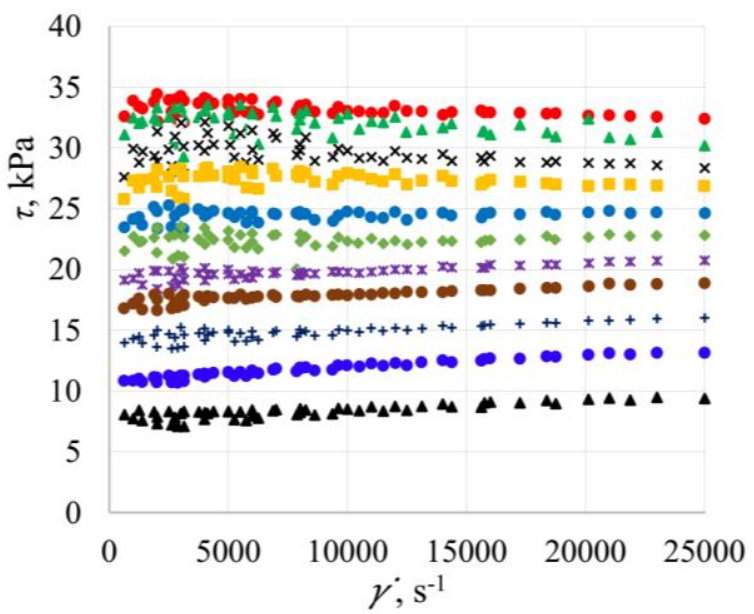

(e)

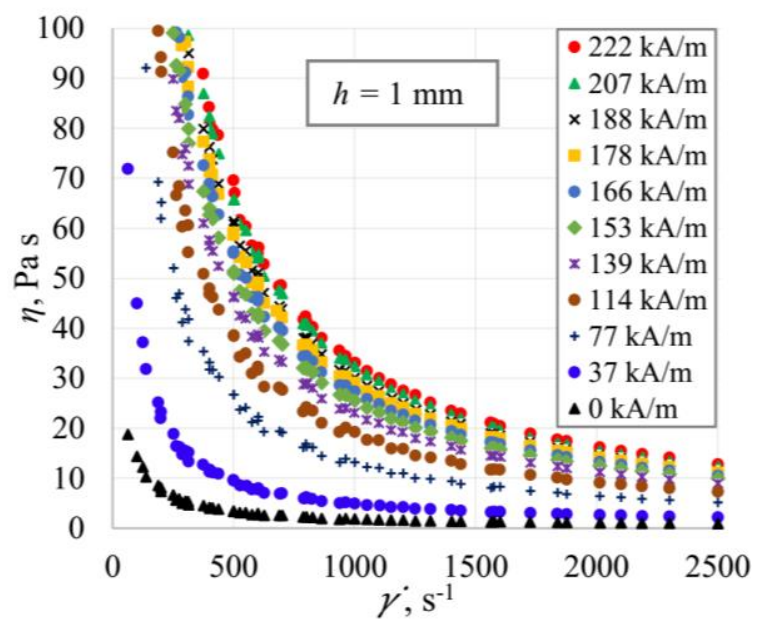

(b)

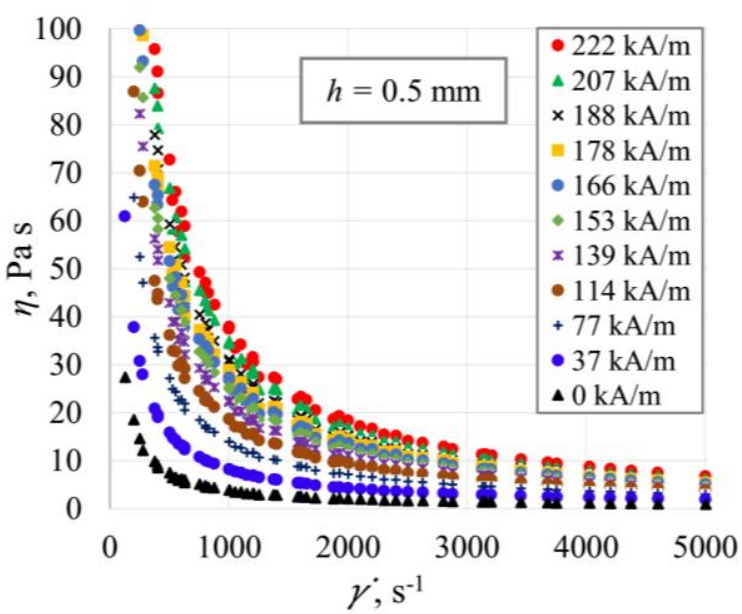

(d)

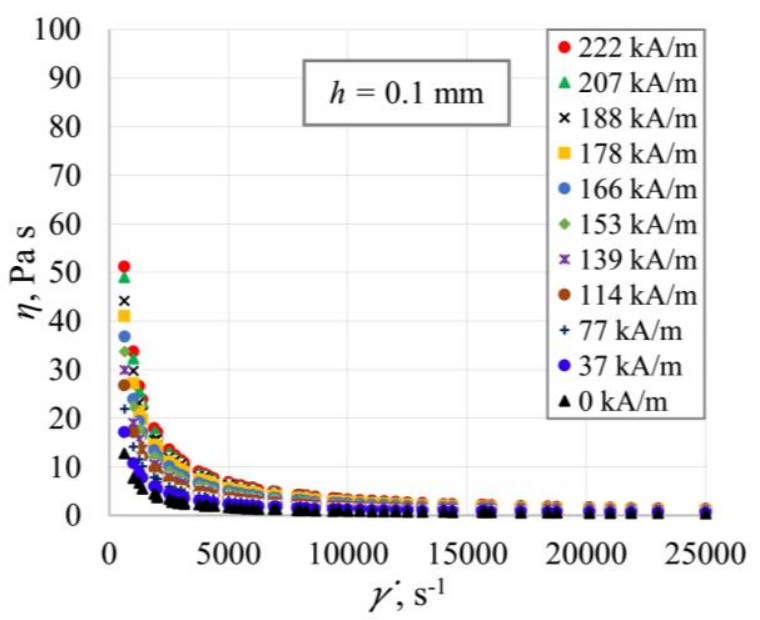

(f)

Figure 5. Shear stress $\tau$ and apparent viscosity $\eta$, as a function of the shear rate $\dot{\gamma}$, for $h=1 \mathrm{~mm}$ (a- 
The flattening curve was also found in [25], where it was hypothesized that high shear rate interferes

$$
\eta=K \dot{\gamma}^{(n-1)}
$$
curves. with the process of forming the particle columns, causing a decrease in the value of the shear stress function of the magnetic field. From the model in [25] a critical shear rate value is found below which the linear correlation between shear rate and shear stress holds. Above the threshold, value there is a reduced number of active particle columns and hence a slight decrease correspondence between shear stress and shear rate. Guth et al. $[25,38]$ also suggested that the high speeds are also responsible for a weakening of the magnetic field in the activation gap, although this is not proven experimentally. In order to overcome the problem of the particular conformation of the flow curves and to obtain a better interpretation of the experimental data, we decided to deepen the analysis of the apparent viscosity

We tried to apply the standard Herschel-Bulkley model, commonly used in the MR fluid characterization, $[39,40]$, but we found that these data fit best an exponential law, rather than the Herschel-Bulkley one. It is thus possible to obtain characteristic parameters to quantify the influence of the high shear rate and the gap thickness on the torque response of the MR fluid. Figure 6a shows that the gap influence is visible for magnetic field values less than or equal to $37 \mathrm{kA} / \mathrm{m}$. For values above 77 $k A / m$ (Figure 6b), the apparent viscosity trends are almost perfectly superimposed and there is no appreciable influence on the gap.

For a first assessment of the influence of gap thickness, we applied the simplified model of Oswald and Waele [24], [25], which was originally built for non-Newtonian fluids:

Where $K$ represents the consistency of the fluid and $n$ is the flow index.

According to this law the shear stress present in the Herschel-Bulkley model is included in the overall shear stress, i.e. the magnetic and the viscous contribution are not separated anymore. We applied to these data a simple regression using Matlab in order to obtain the parameters $K$ and $n$ which best fit the experimental data, as reported in Table 1. 


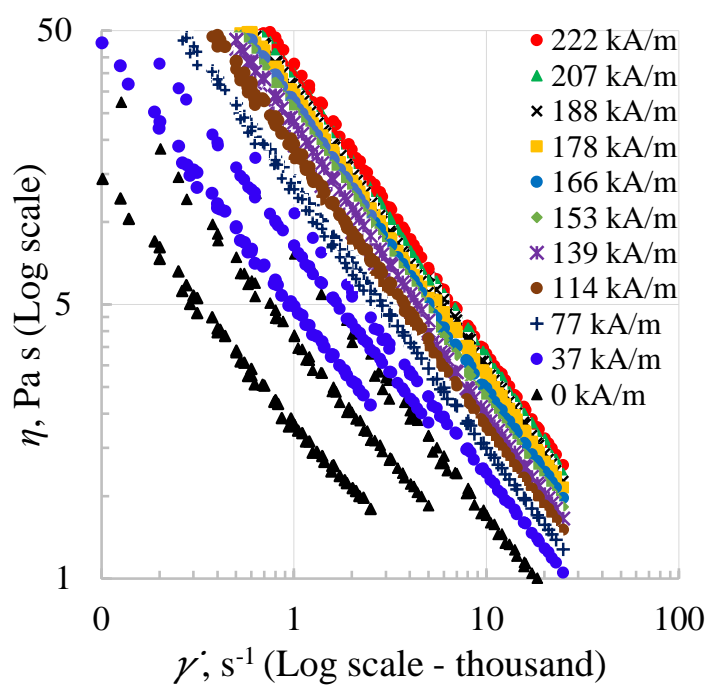

(a)

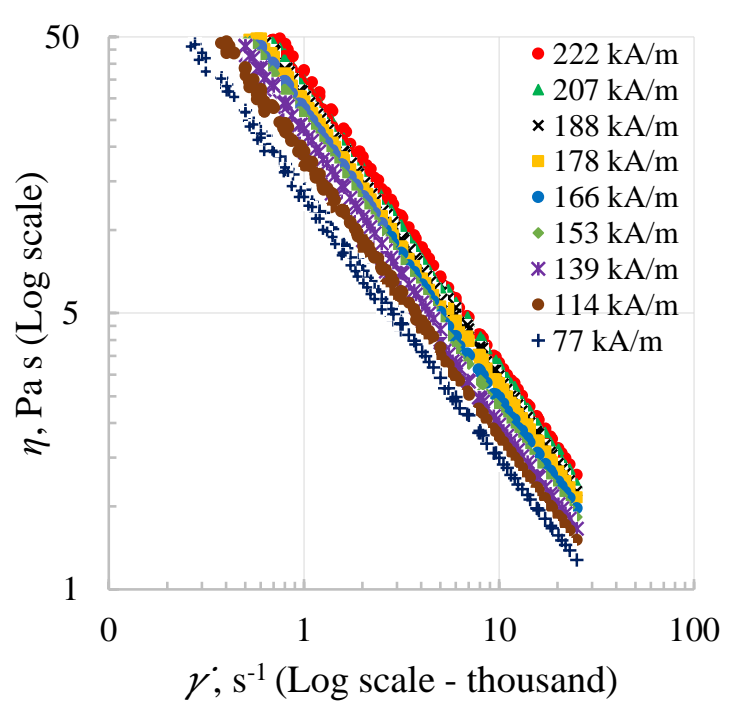

(b)

Figure $6 . \log$-Log diagram of the apparent viscosity $\eta$ vs shear rate $\dot{\gamma}$, for all the gaps combined

(a) and considering only magnetic field above $77 \mathrm{kA} / \mathrm{m}$ (b).

2

Once the values of $K$ and $n$ were determined, it was possible to make a correlation between these data and the input variables. Figure 7 depicts $K$ and $n$ as a function of the applied magnetic field, for each of the three gaps used. The consistency parameter grows almost linearly with the magnitude of the magnetic field, while the magnetic field does not particularly affect the flow index variation. Even the gap thickness seems almost independent of the flow index, while the consistency $K$, for null or very low values $(37 \mathrm{kA} / \mathrm{m})$, grows with the gap thickness decrease. This variation is quite evident at no field, where it passes from about $663 \mathrm{~Pa} \cdot \mathrm{s}^{n}$, with $h=1 \mathrm{~mm}$, to about $7900 \mathrm{~Pa} \cdot \mathrm{s}^{n}$, with $h=0.1 \mathrm{~mm}$. The variation of $K$ at $37 \mathrm{kA} / \mathrm{m}$, is still important, but then fades at higher $H$. These results suggest a relevant influence of the thickness $h$ on the fluid-dynamic phenomena within the gap only at low magnetic field. Then, when the field is applied, the magnetic forces are predominant, masking this dependence.

In order to obtain a simplified model of fluid, it is useful to parameterize the Oswald de Waele equation according to the applied magnetic field. The only parameter to be influenced by $H$ is the consistency $K$, which shows a strong linearity. The flow index seems quite independent on the magnetic field and on the gap, so we simply decided to use its average $\bar{n}=0.02272$. Once the flow index is set, we 
1 recomputed the $K$ value using Matlab and retrieved the following linear relationship, which holds only

2 for the Lord MR 132-DG fluid:

3

$$
\eta_{\text {OdW }}=K(H) \dot{\gamma}^{(\bar{n}-1)}=(118 H+2700) \dot{\gamma}^{(0.2272-1)}
$$

4

$5 \quad$ which is valid using the following units: $H(\mathrm{kA} / \mathrm{m}), \dot{\gamma}\left(\mathrm{s}^{-1}\right), \eta(\mathrm{Pa} \cdot \mathrm{s})$.

Table 1. Ostwald de Waele parameters based on the experimental data.

\begin{tabular}{|c|c|c|c|c|c|c|}
\hline \multirow[b]{2}{*}{$\begin{array}{c}H \\
(\mathrm{kA} / \mathrm{m})\end{array}$} & \multicolumn{2}{|l|}{$h=1 \mathrm{~mm}$} & \multicolumn{2}{|l|}{$h=0.5 \mathrm{~mm}$} & \multicolumn{2}{|c|}{$h=0.1 \mathrm{~mm}$} \\
\hline & $K,\left(\mathrm{~Pa} \mathrm{~s}^{\mathrm{n}}\right)$ & $\mathrm{n}$ & $K,\left(\mathrm{~Pa} \mathrm{~s}^{\mathrm{n}}\right)$ & $\mathrm{n}$ & $K,\left(\mathrm{~Pa} \mathrm{~s}^{\mathrm{n}}\right)$ & $\mathrm{n}$ \\
\hline 222 & 28659.44 & 0.02256 & 31610.48 & 0.01965 & 30867.49 & 0.00996 \\
\hline 207 & 27484.07 & 0.02648 & 29266.51 & 0.01810 & 28468.53 & 0.01557 \\
\hline 188 & 24948.89 & 0.03018 & 25623.56 & 0.02097 & 22182.33 & 0.03709 \\
\hline 178 & 24432.38 & 0.02727 & 22744.66 & 0.02853 & 21644.3 & 0.02950 \\
\hline 166 & 22844.86 & 0.02801 & 20470.62 & 0.03652 & 20873.42 & 0.01916 \\
\hline 153 & 21564.19 & 0.02578 & 19878.36 & 0.02945 & 19652 & 0.01623 \\
\hline 139 & 20219.8 & 0.01864 & 18494.73 & 0.02291 & 17758.69 & 0.01141 \\
\hline 114 & 17478.5 & 0.01116 & 14377.38 & 0.03521 & 14495.21 & 0.02338 \\
\hline 77 & 12604.69 & 0.00327 & 10556.79 & 0.03836 & 12268.82 & 0.02047 \\
\hline 37 & 4176.671 & 0.01733 & 6590.102 & 0.02931 & 9033.781 & 0.02686 \\
\hline 0 & 663.5475 & 0.01554 & 2949.679 & 0.03456 & 7923.72 & 0.00045 \\
\hline
\end{tabular}

This proposed model described by Equation (5), was compared with all the set of experimental data available and the statistical coefficient of determination $R^{2}$ is computed to verify the goodness of the model [41]. The results are depicted in Figure 8, for all the set of data and summarized in Figure 9. The first nine charts, Figure 8a-i shows all the experimental points, which lie almost on a single line, coincident with the proposed model. 


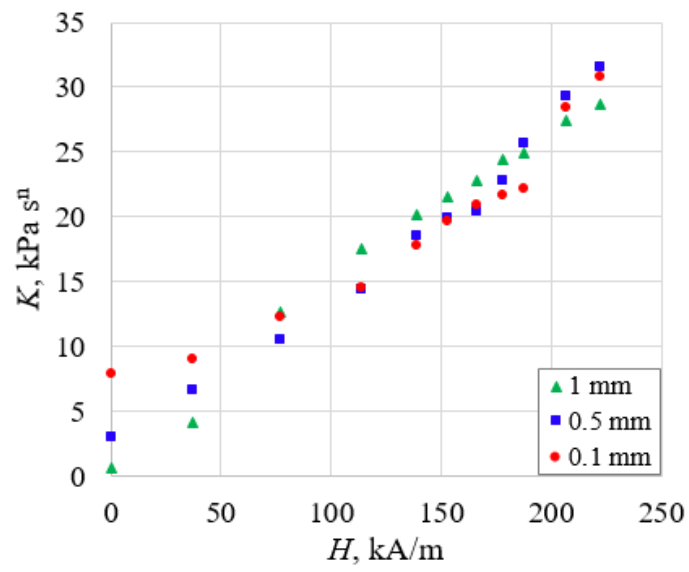

(a)

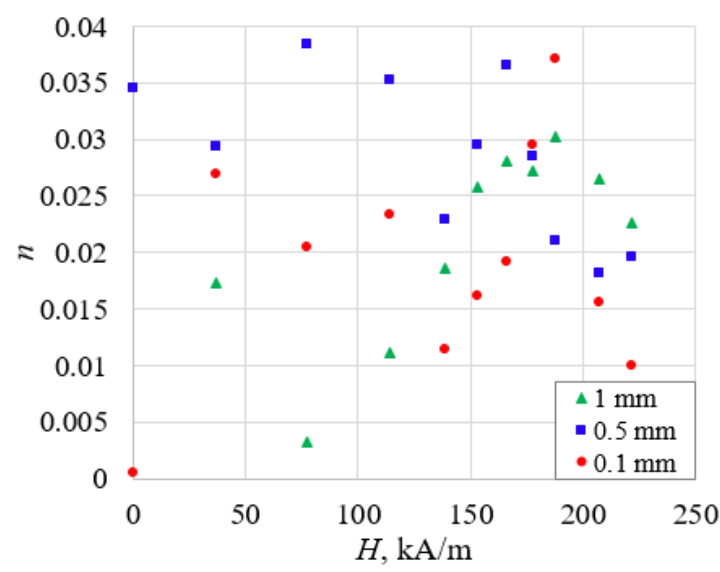

(b)

Figure 7. Correlation of the consistency $K$ (a) and flow index $n$ with the applied magnetic field

The last two charts Figure 81-m are different, the three gaps tested produces a set of data quite different from the other and the model is not able to predict the system behaviour correctly without the information about the gap. Even though the model perfectly fit ( $R^{2}$ greater than 0.99$)$ almost all the data when the magnetic field is above $77 \mathrm{kA} / \mathrm{m}$ while there is a very large error below this value. This confirms that for sufficiently high fields, the fluid can be modelled using a single set of parameters even using different thickness gaps, while nulls of different thicknesses involve different pairs of parameters to describe the MR fluid. The proposed model is valid only for a particular MR fluid, but is interesting to notice two important findings. The off-state behaviour (null or low MR field) can be compared and analysed as if the fluid were a non-Newtonian non-intelligent fluid, which means that the gap thickness and the fluid dynamic will play an important role in the system design, both at low and high shear rate. The on-state behaviour is on the contrary dominated by the presence of strong magnetic fields, which mask the viscous effect and drive the system design. Therefore, it is possible to design a MR system considering separately the two conditions, with a strong advantage in terms of behaviour prediction. 


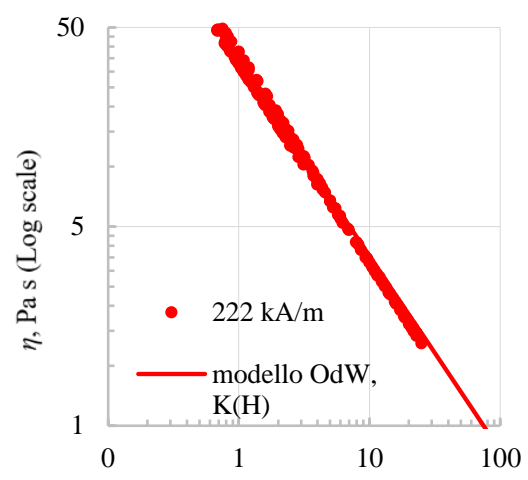

$\gamma^{\prime}, \mathrm{s}^{-1}(\log$ scale - thousand)

(a)

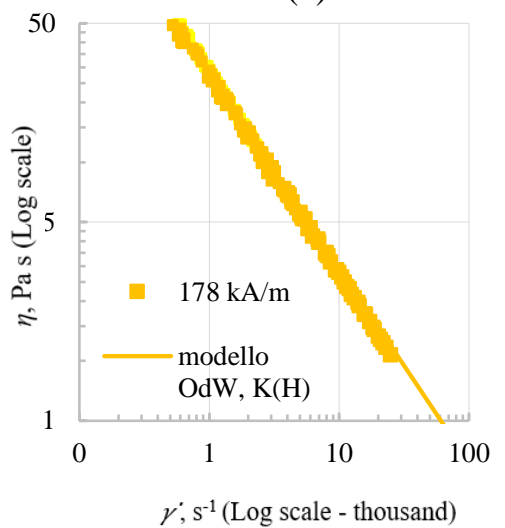

(d)

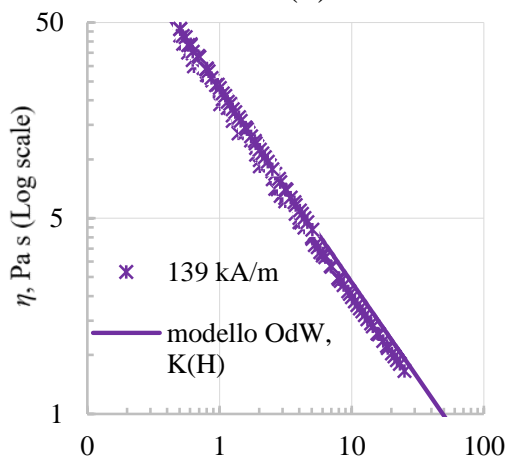

$\gamma^{\prime}, \mathrm{s}^{-1}$ (Log scale - thousand)

(g)

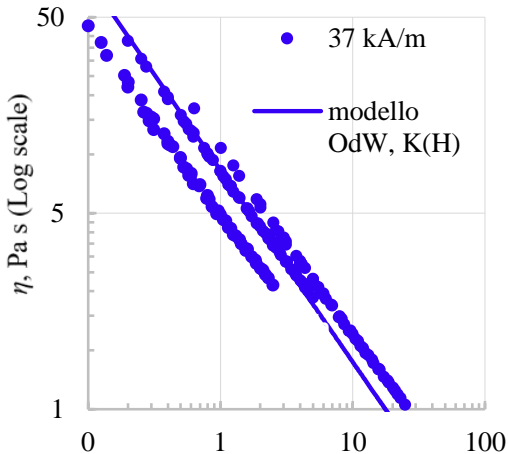

$\gamma^{\prime}, \mathrm{s}^{-1}(\log$ scale - thousand

(1)

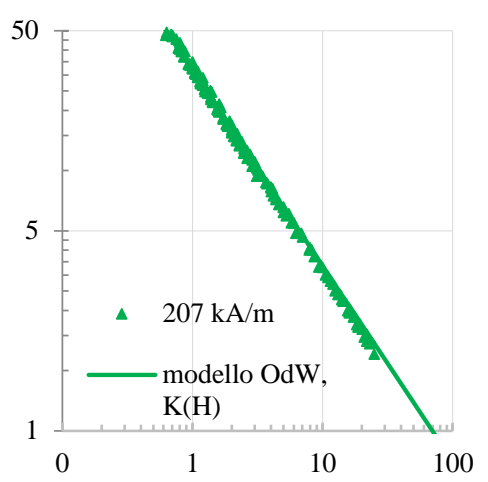

$\gamma^{\prime}, \mathrm{s}^{-1}$ (Log scale - thousand)

(b)

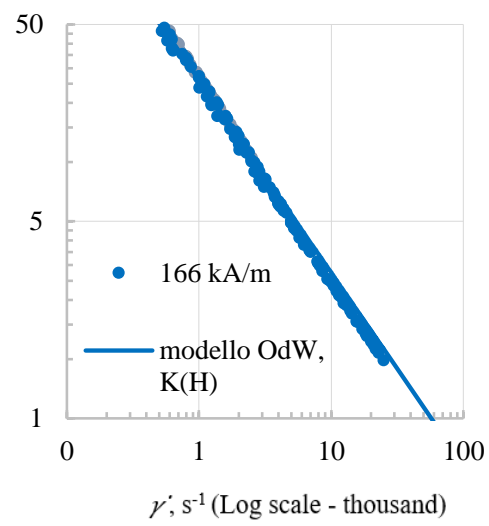

(e)

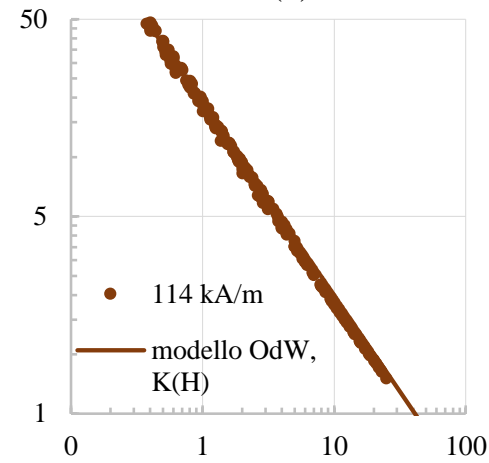

$\gamma^{\prime}, \mathrm{s}^{-1}($ Log scale - thousand)

(h)

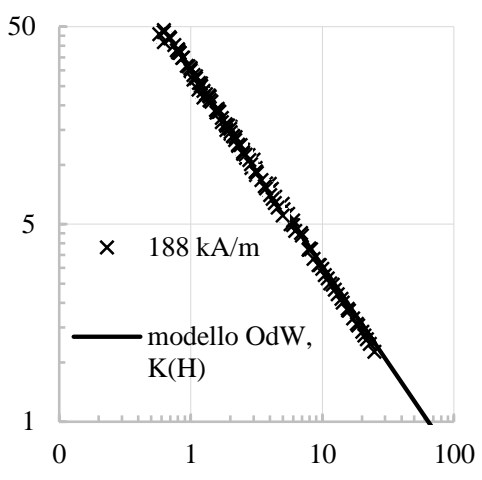

$\gamma^{\prime}, \mathrm{s}^{-1}(\log$ scale - thousand $)$

(c)

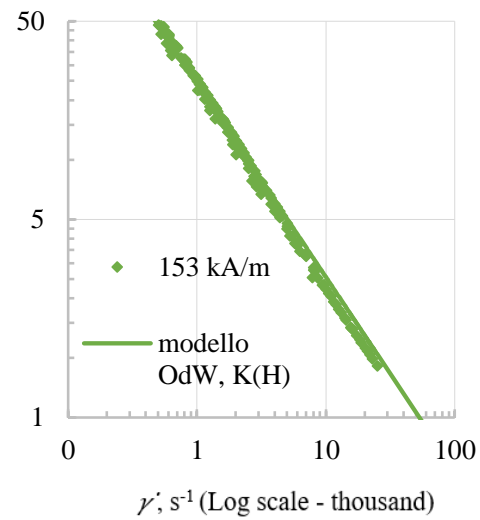

(f)

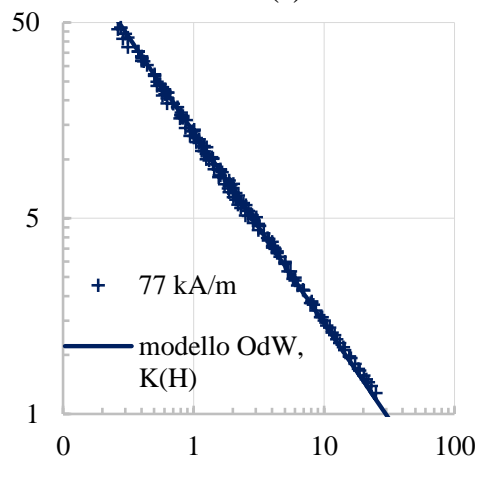

$\gamma^{\prime}, \mathrm{s}^{-1}$ (Log scale - thousand)

(i)

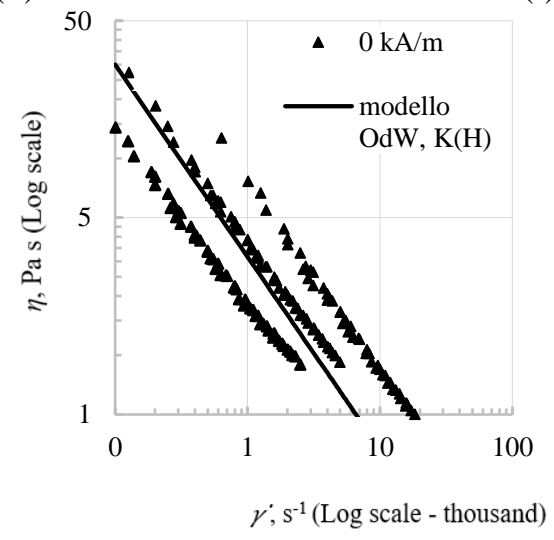

(m)

Figure 8. Comparison between experimental data (dots) and proposed model (solid lines) 


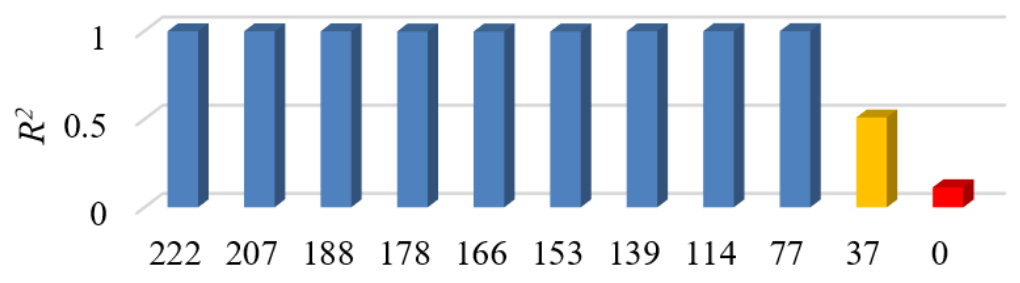

$\mathrm{H}, \mathrm{kA} / \mathrm{m}$

Figure 9. Coefficient of determination $R^{2}$ as a function of the magnetic field $H$. The correlation is very good a part from the two last low magnetic field considered.

\section{CONCLUSION}

This paper analyses the behaviour of a MR fluids at high shear rates and in particular the effect of the gap on the modelling of the fluid, mainly investigating its effect on the shear stress and apparent viscosity. Three gaps were considered at multiple magnetic fields and shear rates. From an extended set of almost two hundred experimental flow curves, we noticed a quite low variation of the flow curves at very high shear rates and magnetic field. From quantitative analyses of the apparent viscosity a set of parameters for the apparent viscosity are retrieved by using a literature model for non-Newtonian fluids, known as Ostwald de Waele. It is possible to simplify the parameter correlation by making the following considerations: the consistency of the model depends only on the magnetic field, the flow index depends on the fluid type and the gap shows an important effect only at null or very low (below $77 \mathrm{kA} / \mathrm{m}$ ) magnetic fields.

This lead to a very useful approach especially in the design phase of a MR based product. During the off state it is possible to disregard the magnetic effect and use a standard viscous model for nonNewtonian fluid, which considers the effect of the gap. This model can be calibrated using a standard rheometer able test the fluid at the desired gap, since no magnetic field is needed. During the active state, the viscous forces are not prevalent even at very high shear rates and the magnetic field dominates the apparent viscosity change, while the gap does not play any significant role. 


\section{ACKNOWLEDGMENTS}

The authors want to acknowledge Professor Norman M. Wereley for the access to the lab, to the equipment and for the fruitful discussion and mentorship in College Park.

\section{REFERENCES}

[1] A. Spaggiari, D. Castagnetti, N. Golinelli, E. Dragoni, G. Scire Mammano, Smart materials: Properties, design and mechatronic applications, Proc. Inst. Mech. Eng. Part L J. Mater. Des. Appl. 0 (2016) 1-29. doi:10.1177/1464420716673671.

[2] J.D. Carlson, D.M. Catanzarite, K.A. St. Clair, COMMERCIAL MAGNETO-RHEOLOGICAL FLUID DEVICES, Int. J. Mod. Phys. B. 10 (1996) 2857-2865. doi:10.1142/S0217979296001306.

[3] S.-B. Choi, W. Li, M. Yu, H. Du, J. Fu, P.X. Do, State of the art of control schemes for smart systems featuring magneto-rheological materials, Smart Mater. Struct. 25 (2016) 43001. doi:10.1088/0964-1726/25/4/043001.

[4] X. Tang, H. Du, S. Sun, D. Ning, Z. Xing, W. Li, Takagi-Sugeno Fuzzy Control for Semi-Active Vehicle Suspension With a Magnetorheological Damper and Experimental Validation, IEEE/ASME Trans. Mechatronics. 22 (2017) 291-300. doi:10.1109/TMECH.2016.2619361.

[5] Z.D. Hu, H. Yan, H.Z. Qiu, P. Zhang, Q. Liu, Friction and wear of magnetorheological fluid under magnetic field, Wear. 278 (2012) 48-52. doi:10.1016/j.wear.2012.01.006.

[6] S.A. Mazlan, N.B. Ekreem, A.G. Olabi, An investigation of the behaviour of magnetorheological fluids in compression mode, J. Mater. Process. Technol. 201 (2008) 780-785. doi:10.1016/j.jmatprotec.2007.11.257.

[7] S.A. Mazlan, A. Issa, H.A. Chowdhury, A.G. Olabi, Tensile Stress-Strain Relationships of Magnetorheological Fluids under Various Factors, Solid State Phenom. 154 (2009) 127-132. doi:10.4028/www.scientific.net/SSP.154.127.

[8] A. Spaggiari, E. Dragoni, Enhanced properties of magnetorheological fluids: Effect of pressure, J. Intell. Mater. Syst. Struct. 26 (2015) 1764-1775. doi:10.1177/1045389X15571386. 
1 [9] A. Spaggiari, E. Dragoni, Effect of pressure on the physical properties of magnetorheological fluids, 23 (2012) 75-86. doi:10.3221/IGF-ESIS.23.08.

[10] A.C. Becnel, N.M. Wereley, Demonstration of Combined Shear and Squeeze Strengthening Modes in a Searle-Type Magnetorheometer, in: Vol. 1 Dev. Charact. Multifunct. Mater. Model. Simul. Control Adapt. Syst. Integr. Syst. Des. Implement., ASME, New York, NY, USA, 2013: p. V001T03A036. doi:10.1115/SMASIS2013-3244.

[11] X.L.Z. Zhang, X.L. Gong, P.Q. Zhang, Q.M. Wang, Study on the mechanism of the squeezestrengthen effect in magnetorheological fluids, J. Appl. Phys. 96 (2004) 2359. doi:10.1063/1.1773379.

[12] P. Kulkarni, C. Ciocanel, S. Vieira, Study of the behavior of MR fluids in squeeze, torsional and

[13] A. Farjoud, R. Cavey, M. Ahmadian, C. Namuduri, Non-dimensional modeling and experimental evaluation of a MR squeeze mode rheometer, J. Phys. Conf. Ser. 149 (2009) 12048. doi:10.1088/1742-6596/149/1/012048.

[14] R. Tao, Super-strong magnetorheological fluids, J. Phys. Condens. MATTER. 13 (2001) 979999.

[15] J.-P. Lucking Bigue, F. Charron, J.-S. Plante, Understanding the super-strong behavior of magnetorheological fluid in simultaneous squeeze-shear with the Peclet number, J. Intell. Mater. Syst. Struct. 26 (2015) 1844-1855. doi:10.1177/1045389X15577657.

[16] J.-P. Lucking Bigue, F. Charron, J.-S. Plante, Understanding the super-strong behavior of magnetorheological fluid in simultaneous squeeze-shear with the Peclet number, J. Intell. Mater. Syst. Struct. 26 (2015) 1844-1855. doi:10.1177/1045389X15577657.

[17] C. Hegger, J. Maas, Investigation of the squeeze strengthening effect in shear mode, J. Intell. Mater. Syst. Struct. (2015) 1045389X15606998-. doi:10.1177/1045389X15606998.

[18] X. Tang, X. Zhang, R. Tao, Y. Rong, Structure-enhanced yield stress of magnetorheological fluids, J. Appl. Phys. 87 (2000) 2634. doi:10.1063/1.372229.

9] N. Sims, R. Stanway, A. Johnson, Vibration isolation using a magnetorheological damper in the 
squeeze-flow

mode,

Proc.

SPIE.

3668

(1999)

520-526.

http://link.aip.org/link/?PSISDG/3668/520/1 (accessed August 3, 2011).

[20] N. Golinelli, A.C. Becnel, A. Spaggiari, N.M. Wereley, Experimental Characterization of Magnetorheological Fluids Using a Custom Searle Magnetorheometer: Influence of the Rotor Shape, IEEE Trans. Magn. 52 (2016) 1-4. doi:10.1109/TMAG.2016.2515983.

[21] J.C. Ulicny, M.A. Golden, C.S. Namuduri, D.J. Klingenberg, Transient response of magnetorheological fluids: Shear flow between concentric cylinders, J. Rheol. (N. Y. N. Y). 49 (2005) 87. doi:10.1122/1.1803576.

[22] G. Schramm, A Practical Approach to Rheology and Rheometry, Gebrueder Haake, 1994.

[23] D. Wang, B. Zi, Y. Zeng, F. Xie, Y. Hou, Measurement of temperature-dependent mechanical properties of magnetorheological fluids using a parallel disk shear stress testing device, Proc. Inst. Mech. Eng. Part C J. Mech. Eng. Sci. 231 (2017) 1725-1737. doi:10.1177/0954406215621099.

[24] D. Wang, B. Zi, S. Qian, J. Qian, Steady-State Heat-Flow Coupling Field of a High-Power Magnetorheological Fluid Clutch Utilizing Liquid Cooling, J. Fluids Eng. 139 (2017) 111105. doi:10.1115/1.4037171.

[25] D. Guth, J. Maas, Characterization and modeling of the behavior of magnetorheological fluids at high shear rates in rotational systems, J. Intell. Mater. Syst. Struct. 27 (2016) 689-704. doi:10.1177/1045389X15577646.

[26] J.D. Carlson, M.R. Jolly, MR fluid, foam and elastomer devices, Mechatronics. 10 (2000) 555569. doi:10.1016/S0957-4158(99)00064-1.

[27] X. Wang, F. Gordaninejad, Flow Analysis of Field-Controllable, Electro- and MagnetoRheological Fluids Using Herschel-Bulkley Model, J. Intell. Mater. Syst. Struct. 10 (1999) 601608. doi:10.1106/P4FL-L1EL-YFLJ-BTRE.

[28] Lord Corporation, MRF-132DG Magneto-Rheological Fluid, 54 (2011) 11. doi:)D DS7015 (Rev.1 11/11).

[29] A.C. Becnel, S.G. Sherman, W. Hu, N.M. Wereley, Squeeze strengthening of 
magnetorheological fluids using mixed mode operation, J. Appl. Phys. 117 (2015) 17C708. doi:10.1063/1.4907603.

[30] A.C. Becnel, N.M. Wereley, Demonstration of Combined Shear and Squeeze Strengthening Modes in a Searle-Type Magnetorheometer, in: Vol. 1 Dev. Charact. Multifunct. Mater. Model. Simul. Control Adapt. Syst. Integr. Syst. Des. Implement., ASME, 2013: p. V001T03A036. doi:10.1115/SMASIS2013-3244.

[31] G.A. Davies, J.R. Stokes, On the gap error in parallel plate rheometry that arises from the presence of air when zeroing the gap, J. Rheol. (N. Y. N. Y). 49 (2005) 919-922. doi:10.1122/1.1942501.

[32] O. Kravchuk, J.R. Stokes, Review of algorithms for estimating the gap error correction in narrow gap parallel plate rheology, J. Rheol. (N. Y. N. Y). 57 (2013) 365-375. doi:10.1122/1.4774323.

[33] G.A. Davies, J.R. Stokes, Thin film and high shear rheology of multiphase complex fluids, J. Nonnewton. Fluid Mech. 148 (2008) 73-87. doi:10.1016/J.JNNFM.2007.04.013.

[34] M.T. López-López, L. Rodríguez-Arco, P. Kuzhir, J.D.G. Duran, A. Zubarev, G. Bossis, StickSlip Instabilities in Magnetorheological Fluids, in: Rheol. Non-Spherical Part. Suspens., Elsevier, 2015: pp. 203-233. doi:10.1016/B978-1-78548-036-2.50007-1.

[35] D. Meeker, FEMM 4.2 - Finite Element Method Magnetics Homepage., (2015). http://www.femm.info/Archives/doc/manual42.pdf (accessed October 6, 2016).

[36] F.D. Goncalves, Characterizing the Behavior of Magnetorheological Fluids at High Velocities and High Shear Rates, Virginia Polytechnic Institute and State University, 2005.

[37] W. Nassar, Pre-yield shearing regime of a magnetorheological fluid (MRF), Solid Mechanics Laboratory- École Polytechnique, 2012.

[38] D. Güth, V. Erbis, M. Schamoni, J. Maas, Design and characteristics of MRF-based actuators for torque transmission under influence of high shear rates up to $34,000 \mathrm{~s}^{-1}$, in: W.-H. Liao (Ed.), International Society for Optics and Photonics, 2014: p. 90572P. doi:10.1117/12.2045306.

[39] F.D. GONCALVES, J.-H. KOO, M. AHMADIAN, A review of the state of the art in magnetorheological fluid technologies - Part I: MR fluid and MR fluid models, Shock Vib. Dig. 

38 (n.d.) 203-219. http://cat.inist.fr/?aModele=afficheN\&cpsidt=18006572 (accessed May 30, 2016).

3 [40] X. Wang, F. Gordaninejad, Flow analysis and modeling of field-controllable, electro-and 4 magneto-rheological fluid dampers, J. Appl. Mech. 74 (2007) 13-22.

5

[41] N.R. Draper, H. Smith, Applied regression analysis, Wiley, 1998.

6 